\title{
Fluorescence Study on Dynamics and Structure of Polymer Chain II. Dependence of Intra-macromolecular Photo-crosslinking Reaction of Polymers with Anthryl and Eosinyl Moieties on Number of Crosslinkable Side Chains
}

\author{
Toshinori TORII, ${ }^{\dagger}$ Hideharu UsHIKI, and Kazuyuki HoRIE* \\ Faculty of General Education, Tokyo University of Agriculture and Technology, \\ 3-5-8, Saiwai-cho, Fuchu-si, Tokyo 183, Japan \\ * Faculty of Engineering, University of Tokyo, 7-3-1, Hongo, \\ Bunkyo-ku, Tokyo 113, Japan
}

(Received September 4, 1992)

\begin{abstract}
Photo-crosslinkable polymers with various compositions of photo-crosslinkable anthryl side chains and eosinyl side chains as emissive probes were synthesized. Degassed solutions of the polymers were irradiated with UV-light to induce an intra-macromolecular photo-crosslinking reactions among anthryl side chains. The rotational diffusion coefficient of the eosinyl group and the translational diffusion coefficient of chain segments during the intra-macromolecular photo-crosslinking reaction were evaluated from the data obtained with an emission probe method. The changes in the dimensions of polymer coils associated with the photo-crosslinking reaction were studied by GPC. The intra-macromolecular photo-crosslinking processes of all sample polymers in solutions are divided into two stages from the view points of reaction kinetics and changes in dimensions and dynamics of a polymer chain. Only in the later stage the shrinkages of polymer coils occurs and the rotational mobilities of side chain decrease rapidly for all sample polymers. The translational mobility of chain segments, however, is suppressed gradually in the initial stage and suppressed rapidly in the later stage. The values for the dimensions and dynamics of polymer chain were considered as functions of conversion of the crosslinkable anthryl side chains. The modes of changes in these values are similar to each other for all polymers, regardless of the number of crosslinkable side chains. The photo-crosslinking process is characterized by the mechanism that in the initial stage crosslinks are formed between nearest-neighbor side chains along the polymer contour and crosslinks are formed between remote side chains in the later stage.
\end{abstract}

KEY WORDS Intramolecular Photo-Crosslinking Reaction / Emission

Probe Method / Polymer Chain Dynamics / Dimension of Polymer /

Mechanism of Intra-macromolecular Crosslinking Reaction /

The intra-macromolecular crosslinking reaction is an important subject in the field of polymer reactions. The rates of activationcontrolled reactions of polymers have been reported by many authors ${ }^{1-4}$ and interpreted mainly on the basis of thermodynamically equilibrated fraction or concentration of cyclic conformations having two reactive groups in a proper juxtaposition. For diffusioncontrolled intramolecular reactions between a pair of terminal groups in polymers, the dependence of the rate of the reaction on molecular weight is reported. ${ }^{5-10}$ Shindo et $a .^{11-13}$ studied intra- and inter-molecular photo-crosslinking reactions of polymers containing cinnamoyl groups in solution. They

* Present address: Department of Reaction Chemistry, Faculty of Engineering, University of Tokyo, 7-3-1, Hongo, Bunkyo-ku, Tokyo 113, Japan. 
reported that the quantum yield for the intramolecular photo-crosslinking reaction increased and microgels abundant with intramolecular crosslinks were produced with increase in molecular weight and density of reactive side chains and with decrease in the concentration of the polymer.

The chemical reactivity of a reactive group in a polymer system is distinguished from that of corresponding model system of small molecules. The reaction rate of a reactive group attached to a polymer chain ought to reflect the dynamical motion of the polymer chain in a diffusion-controlled reaction. We attempted to determine the relationship between the reactivity of a reactive group and mobility of a polymer chain in the course of a diffusion-controlled reaction. Since the photo-dimerization of anthryl moiety, used in our work, proceeds via a sandwich excimer state within a very short lifetime $(<20 \mathrm{~ns})$, the photo-dimerization reaction is also a diffusion-controlled reaction. In the previous work, ${ }^{14}$ a photo-crosslinkable polymer containing anthryl and eosinyl moieties was synthesized and we discussed intra-macromolecular photo-crosslinking reactions on the basis of dimensions and dynamics of the polymer chain. The emission probe method was used since it can be regarded as the best tool for characterizing the mobility and dynamical aspects of the polymer chains in the course of such photo-reactions. ${ }^{15}$ In the previous work, ${ }^{14}$ we found out that the intramacromolecular photo-crosslinking reaction of the polymer was divided into two stages from the view points of changes in dimensions and dynamics of the polymer chain. However the effect of the density of crosslinkable side chains on the reaction kinetics was not clear. It was also not clear why the mode of the reaction changes from the initial stage to the later stage. One possible cause would be change in the number of crosslinkable side chains in individual polymer chains.

In the present work, therefore, photo-cross- linkable polymers with various compositions of photo-crosslinkable anthryl side groups and emissive eosinyl side groups were synthesized, and changes in dimensions of polymer coils, the rotational diffusion coefficient of the eosinyl group, and translational diffusion coefficient of chain segments were evaluated for the UV-light-induced intra-macromolecular photo-crosslinking reaction of these polymers in dimethylformamide (DMF) solutions. The mechanism of the changes in the dimensions and dynamics of polymer chains during the photo-reaction is discussed based on the previously proposed short-range reaction model.

\section{EXPERIMENTAL}

\section{Materials}

Low Molecular Weight Model Compounds. Benzyl 9-anthracenecarboxylate (BA) as the model compound for the photo-crosslinkable anthryl side chain and $2^{\prime}, 4^{\prime}, 5^{\prime}, 7^{\prime}$-tetrabromofluorescein benzylester sodiumsalt (benzyl eosinate) as the model compound for the eosinyl side chain as the emissive probe were prepared as previously reported. ${ }^{14}$ Figure 1 represents the chemical structures of the sample polymers and the model compounds.

Photo-crosslinkable Polymers (PCP). The procedures for the preparation of the original copolymer, copoly(styrene/chloromethylstyrene) [copoly(St/CMSt)], without the pendant groups were previously reported. In order to obtain photo-crosslinkable polymers with various compositions of the anthryl and eosinyl side chains, direct estrification was used. $1 \mathrm{~g}$ of the copoly(St/CMSt) was dissolved into $10 \mathrm{ml}$ of $N, N$-dimethylformamide (DMF), then $0.8 \mathrm{~g}$ of $2^{\prime}, 4^{\prime}, 5^{\prime}, 7^{\prime}$-tetrabromofluorescein disodiumsalt (eosin Y) and various amounts $(1.0,0.75,0.25$, and $0.125 \mathrm{~g})$ of sodium-9-anthracenecarboxylate were added to the solutions. The solutions were stirred at room temperature for 6 hours in dark. Solutions were poured into methanol, the 
<smiles>O=C(OCc1ccccc1)c1c2ccccc2cc2ccccc12</smiles>

Benzyl anthracenecarboxylate<smiles>COc1c(Br)cc2c(-c3ccccc3C(=O)O)c3cc(Br)c(=O)c(Br)c-3oc2c1O</smiles>

Benzyl eosinate

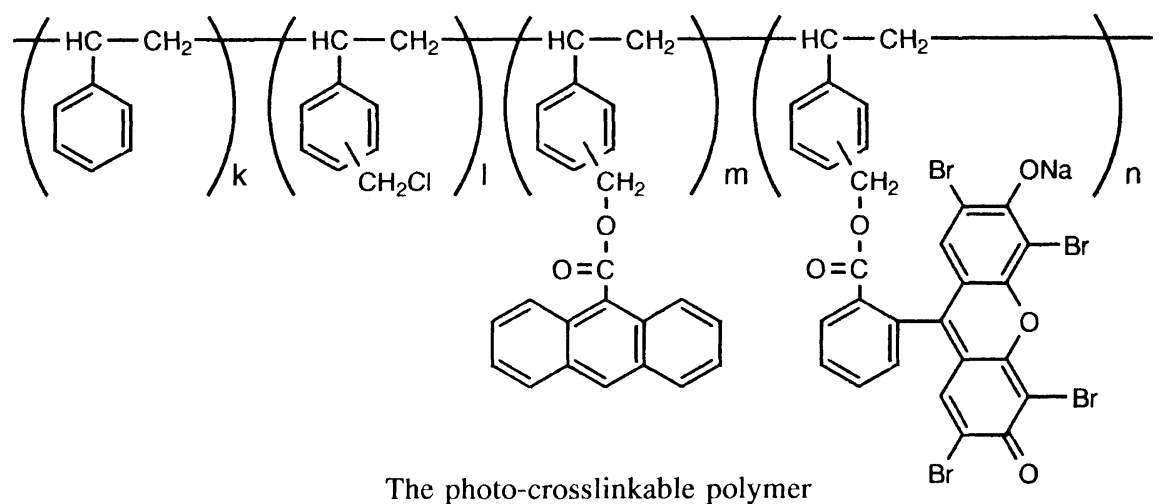

Figure 1. Chemical structures of low molecular weight model compounds and photo-crosslinkable polymers.

Table I. Characteristics of the sample polymers before the intra-macromolecular photo-crosslinking reaction ${ }^{\mathrm{a}}$

\begin{tabular}{lcccc}
\hline Sample polymer & PCP-1 & PCP-2 & PCP-3 & PCP-4 \\
\hline $\mathrm{Mw} / 10^{6}$ & 2.7 & 2.9 & 3.2 & 3.7 \\
$\mathrm{D} . \mathrm{P} . / 10^{4}$ & 2.5 & 2.5 & 3.0 & 3.3 \\
$N_{\mathrm{An}}$ & 1430 & 1330 & 810 & 580 \\
$N_{\text {Eo }}$ & 150 & 180 & 270 & 350 \\
$\left\langle\mathrm{~S}^{2}\right\rangle^{1 / 2} / \mathrm{nm}$ & 60 & 62 & 65 & 72 \\
$c^{*} / \mathrm{gdm}^{-3}$ & 21 & 19 & 18 & 16 \\
$L_{\mathrm{An-An}} / \mathrm{nm}$ & 8.6 & 9.1 & 11 & 14 \\
$L_{\text {Eo-Eo }} / \mathrm{nm}$ & 18 & 18 & 16 & 13 \\
$L_{\mathrm{An}-\mathrm{Eo}} / \mathrm{nm}$ & 8.3 & 8.7 & 10 & 12
\end{tabular}

a $N$, number of chromophores in the individual polymer coil.

$c^{*}$, critical overlap concentration.

$L_{\mathrm{ch}}$, mean distance between chromophores in the polymer coil.

Contents of chloromethylated styrene units are $c a$. $20 \mathrm{~mol} \%$. precipitates were filtered, washed several times with methanol, and dried under vacuum. The resulting photo-crosslinkable polymers with anthryl and eosinyl groups are referred to as PCP-1, $-2,-3$, and -4 , with the order of decrease in the number of anthryl side chains in individual polymer chains. Contents of the photo-crosslinkable anthryl side chains and emissive eosinyl side chains for sample polymers were evaluated from UV-Vis absorption spectra based on molar extinction coefficients of BA and benzyl eosinate as model compounds ${ }^{14}$ for the side chains. Average molecular weights of sample polymers before UV-irradiation were determined with GPC. The results of characterization for sample polymers are summarized in Table I.

Sample Preparation. Each sample polymer 
was dissolved in DMF at a concentration of $0.4 \mathrm{~g} \mathrm{dm}^{-3}$ and $3 \mathrm{ml}$ of the solution was placed in a pyrex cell. In order to minimize photooxidation of anthryl moieties and quenching by oxygen, they were degassed by several freeze-pump-thaw cycles and sealed under high vacuum.

\section{Instruments}

The degassed sample solutions were irradiated with UV-light $(320 \mathrm{~nm}<\lambda<420 \mathrm{~nm})$ by a super high-pressure mercury lamp (Toshiba SHL-100UV) using two glass filters (Toshiba UV-35 and UVD-35) to minimize the dissociation of crosslinks formed in the course of the reaction. The intensity of irradiation light, $I_{0}$, was determined as $2.9 \times 10^{-7}$ einstein . $\mathrm{s}^{-1} \mathrm{~cm}^{-2}$. Spectroscopic and GPC measurements were carried out as reported previously. ${ }^{14}$ All obtained data were stored and analyzed by a personal computer (NEC PC9801) with program softwares prepared at our laboratory.

\section{RESULTS AND DISCUSSION}

\section{Model Compounds.}

Changes in UV-Vis absorption spectra of DMF solution containing two model compounds during UV-irradiation were reported previously. ${ }^{14}$ The second-order plot for the photo-dimerization of benzyl 9-anthracenecarboxylate (BA), on the assumption of proportionality of the concentration of excited state BA to that of ground state BA, failed to give a straight line because of the large absorbance.

The rate of change in concentration of the excited state $\mathrm{BA},\left[\mathrm{BA}^{*}\right]$, under the steadystate irradiation is described by

$$
\begin{aligned}
\mathrm{d}\left[\mathrm{BA}^{*}\right] / \mathrm{d} t= & I_{0}\left(1-10^{-\mathrm{Abs}}\right)-\left(k_{\mathrm{dim}}[\mathrm{BA}]\right. \\
& \left.+k_{\mathrm{f}}+k_{\mathrm{dis}}+k_{\mathrm{isc}}\right)\left[\mathrm{BA}^{*}\right]
\end{aligned}
$$

where $I_{0}$ is the intensity of irradiation light per unit volume per unit time, Abs is absorbance,
[BA] is the concentration of ground state BA, and $k_{\text {dim }}, k_{\mathrm{f}}, k_{\mathrm{dis}}$, and $k_{\text {isc }}$ are rate constants for the photo-dimerization of BA, fluorescence, disactivation, and inter-system crossing, respectively. Making a steady-state approximation, we can estimate $\left[\mathrm{BA}^{*}\right]$ as

$$
\begin{aligned}
{\left[\mathrm{BA}^{*}\right]=} & I_{0}\left(1-10^{-\mathrm{Abs}}\right) / \\
& \left(k_{\mathrm{dim}}[\mathrm{BA}]+k_{\mathrm{f}}+k_{\mathrm{dis}}+k_{\mathrm{isc}}\right)
\end{aligned}
$$

The photo-dimerization is a bimolecular reaction between BA in the excited state and that in the ground state. So the rate of decrease in the concentration of $\mathrm{BA}$ is expressed as

$$
\begin{aligned}
-\mathrm{d}[\mathrm{BA}] / \mathrm{d} t= & k_{\mathrm{dim}}[\mathrm{BA}] \cdot I_{0}\left(1-10^{-\mathrm{Abs}}\right) / \\
& \left(k_{\mathrm{dim}}[\mathrm{BA}]+k_{\mathrm{f}}+k_{\mathrm{dis}}+k_{\mathrm{isc}}\right)
\end{aligned}
$$

Because the value of $\left(k_{\mathrm{dim}}[\mathrm{BA}]+k_{\mathrm{f}}+k_{\mathrm{dis}}+k_{\mathrm{isc}}\right)$ is the reciprocal of the excited singlet lifetime, $\tau_{\mathrm{s}}$, of $\mathrm{BA}$, the equation is described as follows.

$$
-\mathrm{d}[\mathrm{BA}] / \mathrm{d} t=k_{\mathrm{dim}}[\mathrm{BA}] \cdot I_{0}\left(1-10^{-\mathrm{Abs}}\right) \cdot \tau_{\mathrm{s}}
$$

The values of $-\mathrm{d}[\mathrm{BA}] / \mathrm{d} t$ are obtained from the slopes in the plot of [BA] vs. UVirradiation time. By dividing $-\mathrm{d}[\mathrm{BA}] / \mathrm{d} t$ by $[\mathrm{BA}] I_{0}\left(1-10^{-\mathrm{Abs}}\right) \cdot \tau_{\mathrm{s}}$, we obtain the value of $k_{\mathrm{dim}}$. All decay curves of BA are fitted with single exponential functions, and the excited singlet lifetimes of BA do not change during UV-irradiation and are determined to be $10.6 \pm 0.1 \mathrm{~ns}$. Figure 2 represents $k_{\text {dim }}$ as a function of UV-irradiation time. The plots give

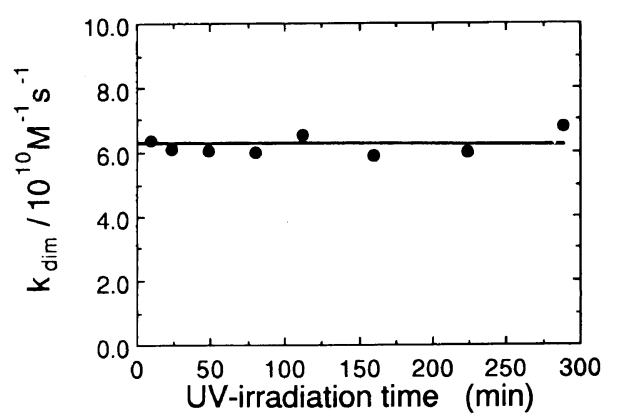

Figure 2. Plot of the rate constant, $k_{\text {dim }}$, for the photodimerization of $\mathrm{BA}$ as a function of UV-irradiation time. 


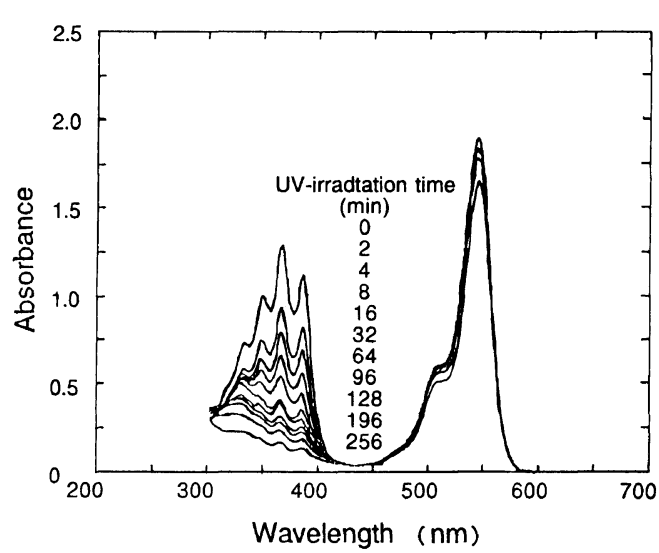

Figure 3. Typical change in UV-Vis absorption spectra of the photo-crosslinkable polymer, PCP-1, in DMF solution during UV-irradiation.

just a constant value of $6.3 \times 10^{10} \mathrm{M}^{-1} \mathrm{~s}^{-1}$ during UV-irradiation. This shows that the photo-dimerization proceeds bimolecularly between excited states $\mathrm{BA}$ and $\mathrm{BA}$ in the ground state.

\section{Polymer Solutions.}

UV-Vis absorption spectra of sample polymer solutions were measured to monitor decrease in the anthryl moiety caused by the photo-crosslinking reaction during UV-irradiation. The change in UV-Vis absorption spectra of PCP-1, the photo-crosslinkable polymer with the largest number of anthryl side chains, during UV-irradiation is shown in Figure 3. The changes in absorbance at $365 \mathrm{~nm}$ corresponding to the anthryl moiety are plotted in Figure 4(a) and changes in conversion of the anthryl moiety are plotted in Figure 4(b) as a function of UV-irradiation time. Large decrease in the anthryl moiety occurs within approximately $40 \mathrm{~min}$ of UVirradiation irrespective of the initial amount of crosslinkable side chains in individual polymer coils. Below here the stage where anthryl moiety decreases rapidly is called the initial stage and the stage where it decreases slowly is called the later stage. No decomposition of dimerized anthryl side chains
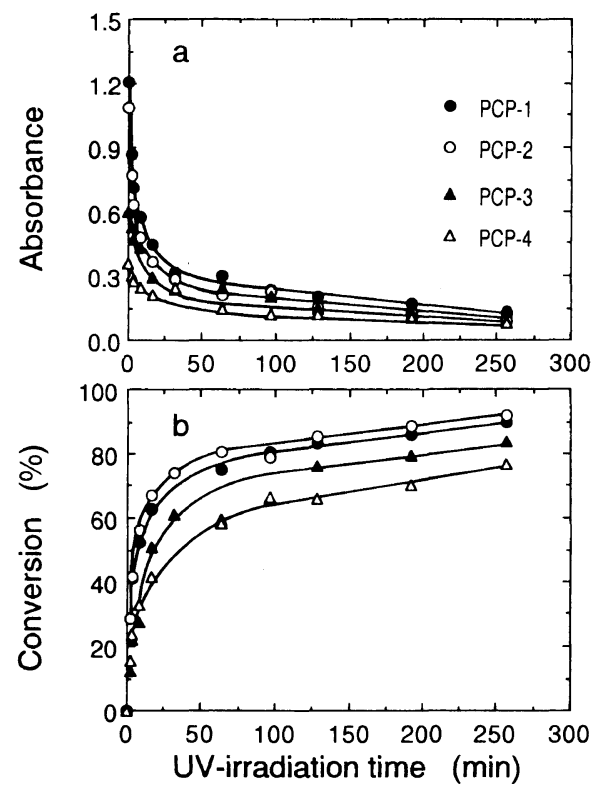

Figure 4. Change in absorbance corresponding to anthryl moiety in the sample polymers; (a), and change in conversion of the anthryl moiety in the sample polymers; (b), against UV-irradiation time.

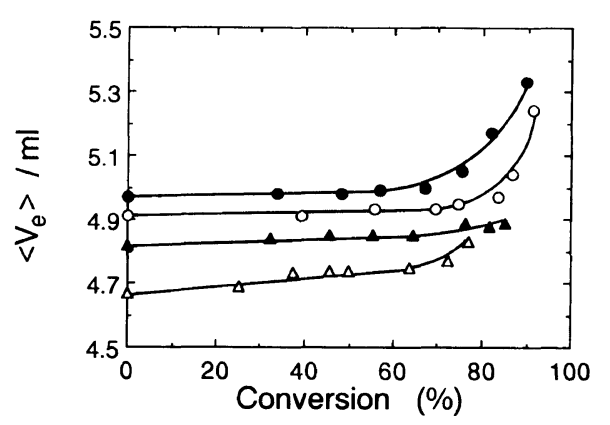

Figure 5. Change in GPC mean elution volume, $\left\langle V_{\mathrm{e}}\right\rangle$, of each polymer against conversion of anthryl moiety in the corresponding sample polymers. PCP-1 (O); PCP-2

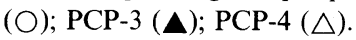

is expected, for the short wavelength light causing the decomposition was cut off.

GPC measurements were carried out for monitoring change in the dimensions of polymer coils during UV-irradiation. Figure 5 depicts changes in mean elution volume, $\left\langle V_{\mathrm{e}}\right\rangle$, as a function of the conversion of the anthryl moiety in corresponding sample polymers. No 


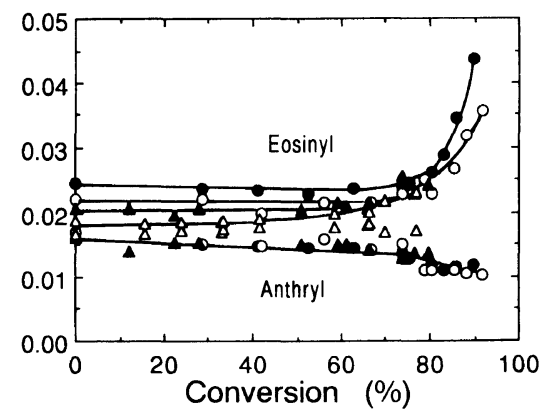

Figure 6. Change in fluorescence anisotropy ratio, $r$, for anthryl and eosinyl moieties in each polymer against conversion of anthryl moiety in the corresponding sample polymers. PCP-1 (O); PCP-2 (○); PCP-3 (A); PCP-4 $(\triangle)$.

remarkable decrease in $\left\langle V_{\mathrm{e}}\right\rangle$, which reflects increase in apparent molecular weight, was observed for any sample polymer. The critical overlap concentration of each sample polymer, $c^{*}$, was calculated on the basis of the initial radius of gyration of the polymer and is summarized in Table I. Since these concentrations of sample polymers $\left(0.4 \mathrm{~g} \mathrm{dm}^{-3}\right)$ are much lower than the values of $c^{*}$, polymer coils do not entangle with each other and the photo-crosslinking reaction is expected to proceed only in individual polymer coils. The values of $\left\langle V_{\mathrm{e}}\right\rangle$ increase only in the later stage showing that polymer coils shrink in the later stage. With increase in the number of crosslinkable side chains in individual polymer coils (PCP-1 and -2), large shrinkage occurs.

To monitor changes in the rotational mobility of side chain associated with structural changes of polymer coils, the fluorescence anisotropy ratio, $r$, was measured for both chromophores by using fluorescence depolarization method. The value of $r$ was calculated from four emission spectra by

$r=\left(I_{\mathrm{VV}}-G I_{\mathrm{VH}}\right) /\left(I_{\mathrm{VV}}+2 G I_{\mathrm{VH}}\right) ; \quad G=I_{\mathrm{HV}} / I_{\mathrm{HH}}$

where $I$ represents the fluorescence intensities and subscripts represent orientation of polarizers ( $V$ is vertical and $H$ is horizontal), which are located at the side of incident light (the

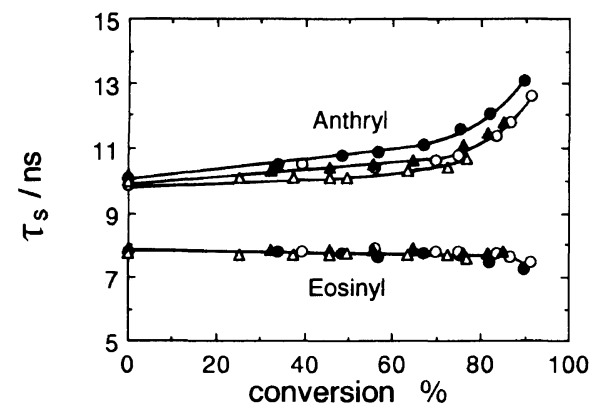

Figure 7. Change in excited singlet life time, $\tau_{s}$, of anthryl and eosinyl moieties in each polymer against conversion of anthryl moiety in the corresponding sample polymers. PCP-1 (O); PCP-2 (○); PCP-3 (A); PCP-4 $(\triangle)$.

first subscript) and at the side of emitted light (the second subscript). $G$ is used for correcting the depolarization characteristics of the apparatus having grating-type monochromators. The fluorescence anisotropy ratios, $r$, for two chromophores are depicted in Figure 6 as a function of the conversion of the anthryl moiety in corresponding sample polymers. The values of $r$ for the eosinyl moiety become large in the later stage for all sample polymers and the extent of the change becomes large with increase in number of anthryl moieties in the polymer coil. But the values of $r$ for anthryl moiety change slightly.

Excited singlet lifetimes, $\tau_{\mathrm{s}}$, of both chromophores were measured with single photon counting method. All decay curves for both chromophores were fitted by single-exponential functions. $\tau_{\mathrm{s}}$ for the anthryl group was used for estimating the translational diffusion coefficient of chain segments based on the energy transfer from the anthryl to the eosinyl group in individual polymer coils. $\tau_{\mathrm{s}}$ for the eosinyl group was used for evaluating the rotational diffusion coefficient of the eosinyl side chain. In Figure 7 , the values of $\tau_{\mathrm{s}}$ for both chromophores are plotted against the conversion of the anthryl moiety in the corresponding sample polymer. The values of $\tau_{\mathrm{s}}$ for the eosinyl group do not change remarkably. Those for the anthryl group become 
longer in the later stage for all sample polymers. The extent of change in $\tau_{\mathrm{s}}$ for the anthryl moiety became large with the increase in the number of anthryl side chains in the polymer. The elongation of $\tau_{\mathrm{s}}$ for the anthryl group during the shinkage of polymer coils shows that quenching to the anthryl emission becomes small with the formation of crosslinks in the later stage. ${ }^{14}$

\section{Analysis.}

Dimensions of Polymer Coils. The relationship between GPC elution volume, $V_{\mathrm{e}}$, and product of intrinsic viscosity and molecular weight, $[\eta] M$, of sample polymer is known to be universal irrespective of nature of samples for a given GPC column. ${ }^{16}$ GPC measurements were carried out with a column whose universal curve was obtained from measurements of poly(styrene) standards. By using the calibration curve, we transform $V_{\mathrm{e}}$ into $[\eta] M$ for the present polymers which were UVirradiated for various period in minutes. From the Flory-Fox's equation, ${ }^{17}$ we obtain

$$
[\eta] M=6^{3 / 2} \Phi_{\mathrm{F}}\left\langle S^{2}\right\rangle^{3 / 2}
$$

where $\left\langle S^{2}\right\rangle^{1 / 2}$ is the mean radius of gyration of polymer coil. For non-draining polymer coils, $\Phi_{\mathrm{F}}$ can be regarded as a constant for the first approximation. The $\left\langle S^{2}\right\rangle^{1 / 2}$ is obtained by substituting the $[\eta] M$ for a sample polymers into eq. 5 .

Rotational Mobility of the Eosinyl Side Chain. Mean distances among chromophores, $L_{\text {ch }}$, before UV-irradiation were calculated from the following equation

$$
L_{\mathrm{ch}}=\left\{(4 \pi / 3) \cdot\left\langle S^{2}\right\rangle^{3 / 2} / N_{\mathrm{ch}}\right\}^{1 / 3}
$$

where $N_{\mathrm{ch}}$ is the number of chromophores in an individual polymer coil. The values of $L_{\mathrm{ch}}$ are summarized in Table I. The mean distance among eosinyl moieties, $L_{\mathrm{Eo}-\mathrm{Eo}}$, was not less than $13 \mathrm{~nm}$. This distance is much longer than the Foerster radius for a general singletsinglet energy transfer (2-5nm). Assuming the absence of Foerser type energy migration among eosinyl side chains, the rotation diffusion coefficient, $D_{\mathrm{r}}$, of the eosinyl moiety in the polymer coil was evaluated using the values of $r$ and $\tau^{\mathrm{s}}$ as follows.

$$
D_{\mathrm{r}}=\left\{1 /\left(6 \tau_{\mathrm{s}}\right)\right\} \cdot\left(r_{0}-r\right) / r
$$

The value of $r_{0}$ for eosinyl side chain was assumed to be the same value for eosin $Y$ $\left(r_{0}=0.29\right)$.

Translational Mobility of Segmental Chain. We consider the nonradiative energy transfer from the anthryl group to the eosinyl group. According to the Stern-Volmer equation, the excited singlet lifetime of an energy donor, $\tau_{\mathrm{s}}$, is related to the rate constant for the energy transfer, $k_{\mathrm{tr}}$, and the concentration, [E], of an energy acceptor by

$$
1 / \tau_{\mathrm{s}}-1 / \tau_{\mathrm{s} 0}=k_{\mathrm{tr}}[\mathrm{E}]
$$

where $\tau_{\mathrm{s} 0}$ is the excited singlet lifetime of the energy donor in the absence of the energy acceptor. The local concentration of the eosinyl moiety as the energy acceptor in individual polymer coil, [E], can be estimated from the value of $\left\langle S^{2}\right\rangle^{1 / 2}$ mentioned above. Then we obtain $k_{\mathrm{tr}}$ by

$$
\begin{aligned}
k_{\mathrm{tr}}= & \left(1 / \tau_{\mathrm{s}}-1 / \tau_{\mathrm{s} 0}\right) /[\mathrm{E}] ; \\
& {[\mathrm{E}]=\left(N_{\mathrm{Eo}} / N_{\mathrm{A}}\right) /\left\{4 \pi / 3 \cdot\left\langle S^{2}\right\rangle^{3 / 2}\right\} }
\end{aligned}
$$

where $N_{\mathrm{Eo}}$ is the mean number of eosinyl moiety in a single polymer coil and $N_{\mathrm{A}}$ is Avogadro's number. The values of $k_{\mathrm{tr}}$ are estimated by using $\tau_{\mathrm{s}}$ of the anthryl group in the polymer and $\tau_{\mathrm{s} 0}=14.5 \mathrm{~ns}$. Since the energy transfer from the anthryl group to the eosinyl group is a bimolecular reaction, the value of translational diffusion coefficient, $D_{\mathrm{s}}$, is evaluated by using the following relation ${ }^{18}$

$$
D_{\mathrm{s}}=k_{\mathrm{tr}} /\left(8 \pi R N_{\mathrm{A}}\right)
$$

where $R$ is the critical radius of the energy transfer and was assumed to be $2 \mathrm{~nm} .{ }^{14}$

Reaction Kinetics. We treat the intra-macromolecular photo-crosslinking reaction as a reaction between anthryl side chain in the excited state and that in the ground state, 
similarly to the case of the photo-dimerization of the anthryl model compound. The local concentration of the anthryl moiety in an individual polymer coil, $\left[\mathrm{An}^{\mathrm{p}}\right]$, is given as eq 11 , using the number of anthryl moieties in the polymer coil, $N_{\mathrm{An}}{ }^{p}$, and volume of the polymer coil, $V_{\mathrm{p}}$,

$$
\left[\mathrm{An}^{\mathrm{p}}\right]=\left(N_{\mathrm{An}}^{p} / N_{\mathrm{A}}\right) / V_{\mathrm{p}} ; \quad V_{\mathrm{p}}=(4 \pi / 3) \cdot\left\langle S^{2}\right\rangle^{3 / 2}
$$

where $N_{\mathrm{A}}$ is Avogadro's number and $\left\langle S^{2}\right\rangle^{1 / 2}$ is the mean radius of gyration of polymer coils estimated from GPC data. The steadystate concentration of excited anthryl group, $\left[\mathrm{An}^{*}\right]$, in the solution is estimated from

$$
[\mathrm{An} *]=I_{0}\left(1-10^{-\mathrm{Abs}}\right) \cdot \tau_{\mathrm{s}}
$$

by using the absorbance, Abs, of the anthryl group and intensity of irradiation light per unit volume per unit time, $I_{0}$. Then we obtain the rate of decrease in the concentration of anthryl moiety in the solution by

$$
-\mathrm{d}[\mathrm{An}] / \mathrm{d} t=k_{\mathrm{c}}\left[\mathrm{An}^{\mathrm{p}}\right] I_{0}\left(1-10^{-\mathrm{Abs}}\right) \cdot \tau_{\mathrm{s}}
$$

where $k_{\mathrm{c}}$ is the rate constant of the photocrosslinking reaction. Similar to the case of the photo-dimerization of BA, the values of $k_{\mathrm{c}}$ are obtained by evaluating the slopes, $-\mathrm{d}[\mathrm{An}] / \mathrm{d} t$, in plots for absorbance vs. UVirradiation time and then dividing the slopes by the values of $\left[\mathrm{An}^{\mathrm{p}}\right] I_{0}\left(1-10^{-\mathrm{Abs}}\right) \cdot \tau_{\mathrm{s}}$. Figure 8 represents the $k_{\mathrm{c}}$ against conversion of the

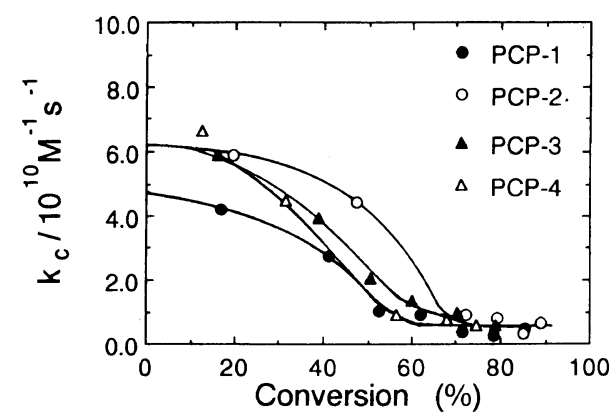

Figure 8. Plots of rate constants, $k_{\mathrm{c}}$, for intra-macromolecular photo-crosslinking reactions as functions of conversion of the anthryl moiety in the corresponding sample polymers. anthryl moiety in the corresponding polymers. Apparently the values of $k_{\mathrm{c}}$ for all sample polymers decrease rapidly in the initial stage.

Changes in Dimension and Mobility of Polymer Chain. In the previous paper, ${ }^{14}$ we discussed the changes in the dimensions and dynamics of a polymer chain as a function of the extent of the reaction of anthryl moiety rather than UV-irradiation time. The rate of the photo-crosslinking reaction itself increases with the number of photo-crosslinkable side chains in sample polymers, as is shown in Figure 4 . The reaction rate is influenced by the initial number of anthryl moieties in the

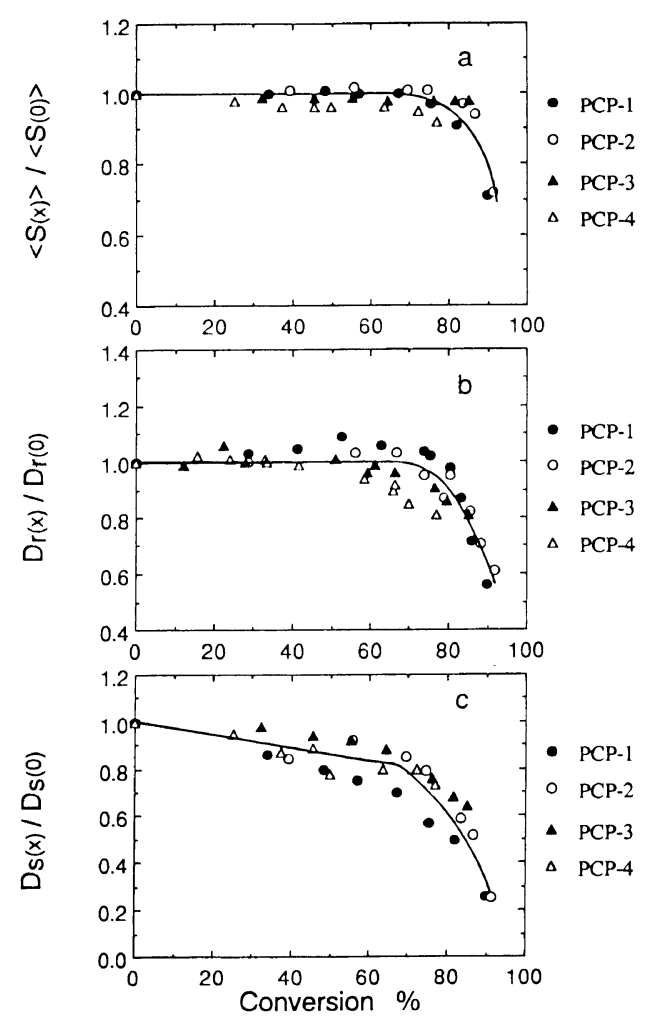

Figure 9. Plots for $\left\langle S_{(x)}\right\rangle /\left\langle S_{(0)}\right\rangle, D_{\left.r^{(x)}\right)} / D_{r^{(0)}}$, and $D_{\left.s^{(x)}\right)}$ $D_{s^{(0)}}$ for sample polymers as functions of conversion of anthryl moiety in the corresponding sample polymers.

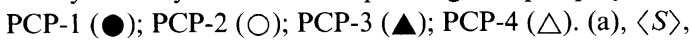
gyration radius of the polymer coils; (b), $D_{r}$, rotational diffusion coefficient of eosinyl moiety in the polymers; (c), $D_{s}$, translational diffusion coefficient of chain segment of the polymers. 
polymers. However the changes in $\left\langle S^{2}\right\rangle^{1 / 2}, D_{\mathrm{r}}$ and $D_{\mathrm{s}}$ during the photo-crosslinking reaction can be discussed more clearly as a function of the conversion of the anthryl moiety, $x$, in corresponding polymers as are shown in Figure 9. These physical parameters change similarly in the course of reaction regardless of the initial number of crosslinkable anthryl side chains and give universal curves.

The relative change in the ratio of the mean radius of gyration at conversion, $x$, to its initial value, $\left\langle S_{(x)}\right\rangle /\left\langle S_{(0)}\right\rangle$, is depicted in Figure 9-a. Remarkable shrinkage of the polymer coils takes place when the conversion exceeds $c a$. $70 \%$. The extent of shrinkage increases with the number of anthryl moieties (Figure 5). PCP-4, with a small number of crosslinkable side chains, shows slight shrinkage in the initial stage. When a crosslink is formed in PCP-4 between nearest-neighbor side chains in the initial stage, a large dangling chain is formed because the distance between these side chains is long. Therefore even the formation of small amounts of crosslinks causes slight shrinkage in PCP-4.

Figure 9-b shows change in $D_{\mathrm{r}}$ at conversion $x, D_{\mathrm{r}^{(x)}}$, relative to its initial value, $D_{\mathrm{r}^{(0)}}$. Remarkable decrease in $D_{\mathrm{r}^{(x)}} / D_{\mathrm{r}^{(0)}}$ occurs when the conversion exceeded $c a$. $70 \%$. For PCP-1 and PCP-2 slight increase in $D_{\mathrm{r}^{(x)}} / D_{\mathrm{r}^{(0)}}$ was observed before the conversion reached the critical value, which correspond to the very slight increase in $\left\langle S_{(x)}\right\rangle /\left\langle S_{(0)}\right\rangle$ for these polymers. Moreover the slight decrease in $D_{\left.\mathrm{r}^{(x)}\right)} / D_{\mathrm{r}^{(0)}}$ for PCP-4 corresponds to the slight decrease in $\left\langle S_{(x)}\right\rangle /\left\langle S_{(0)}\right\rangle$ for the polymer. Thus changes in $D_{\left.\mathrm{r}^{(x)}\right)} / D_{\mathrm{r}^{(0)}}$ and in $\left\langle S_{(x)}\right\rangle /\left\langle S_{(0)}\right\rangle$ agree well, though the values of $D_{\mathrm{r}}$ and $\left\langle S^{2}\right\rangle^{1 / 2}$ have been evaluated independently.

The rotational diffusion coefficient, $D_{\mathrm{r}}$, is related to the volume of the eosinyl moiety as a rotational body, $v_{\text {rot }}$, and the viscosity, $\eta$, of its environment inside the polymer coil by

$$
D_{\mathrm{r}}=k_{\mathrm{B}} T /\left(6 v_{\mathrm{rot}} \eta\right)
$$

where $k_{\mathrm{B}}$ is Boltzman's constant and $T$ is temperature. Because $v_{\text {rot }}$ does not change during the intra-macromolecular photo-crosslinking reaction, the suppression of rotational mobility is attributed to increase in the microviscosity inside the polymer coil. Apparently the increase in the internal viscosity is caused by shrinkage of the polymer coil.

Figure 9-c shows the dependence of $D_{\mathrm{s}}$ at conversion $x, D_{\mathrm{s}^{(x)}}$, relative to its initial value, $D_{\mathrm{s}^{(0)}}$, on the conversion of the anthryl moiety in the corresponding polymer. The values of $D_{\mathrm{s}^{(x)}} / D_{\mathrm{s}^{(0)}}$ decrease gradually in the initial stage. Such gradual decrease, however, was not observed for $D_{\left.\mathrm{r}^{(x)}\right)} / D_{\mathrm{r}^{(0)}}$ and $\left\langle S_{(x)}\right\rangle /\left\langle S_{(0)}\right\rangle$ in the initial stage and these values decrease rapidly only in the later stage. The values of $k_{\mathrm{c}}$ for the intra-macromolecular photo-crosslinking reaction decrease in the initial stage as is shown in Figure $8 . k_{\mathrm{c}}$ is the rate constant for the bimolecular diffusion-controlled reaction between anthryl side chains and $D_{\mathrm{s}}$ is the value estimated based on such the reaction between anthryl and eosinyl groups. Because the photo-dimerization between anthryl side chains requires a very short critical radius of reaction $(\approx 0.4 \mathrm{~nm})$, the formation of a few crosslinks makes the rate constant of the dimerization decrease effectively in the initial stage as shown in Figure 8. In the later stage, further decrease in the rate constant was not clearly observed. Since the Foerster radius of a energy transfer $(2-5 \mathrm{~nm})$ is longer than that of the photo-dimerization, $D_{\mathrm{s}}$ is less sensitive to formation of crosslinks than $k_{\mathrm{c}}$ is. Remarkable decrease in $D_{\mathrm{s}^{(x)} / D_{\mathrm{s}^{(0)}}}$ was observed when the conversion exceeded $c a .70 \%$, which is attributed to increase in the microviscosity inside polymer coil due to shrinkage.

\section{The Mechanism for the Intra-macromolecular}

\section{Photo-crosslinking Reaction}

In the present work, we found that the mode of the intra-macromolecular photocrosslinking reaction changes with the progress of the reaction from the view points of the reaction kinetics, and changes in dimension 


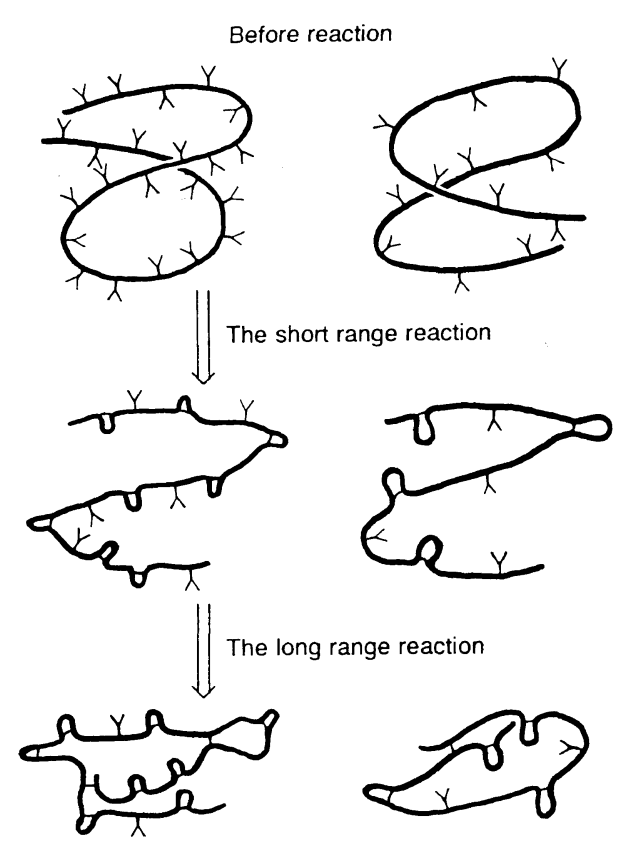

Figure 10. Schematic representation of the process of the intra-macromolecular photo-crosslinking reaction. Photo-crosslinkable side chains are represented by ' $Y$ '.

and dynamics of polymer chain. The rate of the reaction decreases rapidly and the translational mobility of chain segments decreases gradually in the initial stage, irrespective of the initial number of crosslinkable side chains. The dimensions of polymer coils and the rotational mobility of side chain decrease only in the later stage. Remarkable changes in dimensions and dynamics occur when the conversion exceeds $c a$. $70 \%$, which means that "the short range reaction model" ${ }^{14}$ can be applied again to this intra-macromolecular photo-crosslinking reaction regardless of the initial number of crosslinkable side chains. The short range reaction model is described as follows. In the initial stage, crosslinks are formed between nearest-neighbor side chains (the short range reaction) along the polymer chain contour. In this stage, the dimensions of the polymer coils change little and the translational mobility of chain segments is gradually suppressed with the formation of crosslinks and dangling chains. In time, re- active side chains at the nearest neighbor to a certain reactive moiety are consumed by the short range reaction. Then crosslinks are formed between remote side chains which remained unreacted in the initial stage. Polymer coils shrink, the density of monomer units and the micro-viscosity inside the polymer coils increases. Hence rotational and translational mobilities are suppressed in the later stage. Thus, the short range reaction causes increase in the rigidity of chain segments and results in suppression of reactivity. The long range reaction in the later stage causes shrinkage of the polymer coil and suppression of both the rotational mobility of side chains and translational mobility of chain segments.

Theoretical treatment for the reactant isolation was made by Flory $^{19}$ and Cohen et $a l .{ }^{20}$ as a one-dimensional problem. For an one-dimensional polymer chain with an infinite degree of polymerization, the probability of survival of reactive side chains in a reaction between nearest-neighbor side chains is statistically evaluated to be $e^{-2}(13.5 \%)$. Namely $86.5 \%$ of reactive side chains are consumed by this reaction because they treated this problem as an one-dimensional problem where the flexibility of polymer coil was ignored and further reaction was not considered. But the real polymer chain has some flexibility in three-dimensional space, and not only reactive groups in juxtapositions but also those separated by some or more monomer units can react. In the present case, the photocrosslinking reaction occurs between anthryl moieties lying several percents along the polymer, which is quite different from the above one-dimensional case. Nevertheless, the remarkable change of the mode of reaction occur at $c a .70 \%$ conversion. Its deviation from the above-calculated value $(86.5 \%)$ is attributed to the fact that certain amounts of crosslinks are formed by the long range reaction concurrently with the short range reaction and the fraction of crosslinks formed by the long range reaction increases with the 
progress of total reactions. The preferential occurrence of the short range reaction in the initial stage would be caused also by the fact that the present photo-reaction proceeds through excited singlet state with very short lifetime (10-20 ns).

In conclusion, changes in the dimensions and dynamics of polymer chains are not influenced by the total number of crosslinks formed, but by the formation of crosslinks between remote side chains which remained unreacted during the short range reaction. This is the reason why the difference in the number of crosslinkable side chains in the present case does not affect the process of the intra-macromolecular photo-crosslinking reaction. Simultaneous measurements of GPC, fluorescence spectra, fluorescence anisotropy ratio, and excited singlet life time during the intra-macromolecular photo-crosslinking reaction make it possible to discuss the mechanism of the intra-macromolecular photocrosslinking reaction.

\section{REFERENCES AND NOTES}

1. N. Goodman and H. Morawetz, J. Polym. Sci., A, 9, 1657 (1971).

2. M. Sisido, Macromolecules, 4, 737 (1971).
3. M. Sisido, Y. Imanishi, and T. Higashimura, Macromolecules, 9, 316 (1976).

4. J. A. Semlyer, Adv. Polym. Sci., 21, 41 (1976).

5. T. Kanaya, K. Goshiki, M. Yamamoto, and Y. Nishijima, J. Am. Chem. Soc., 104, 3580 (1982).

6. M. A. Winnik, T. Redpath, and D. H. Richards, Macromolecules, 13, 328 (1980).

7. M. A. Winnik, A. E. C. Redpath, K. Paton, and J. Danhelka, Polymer, 25, 91 (1984).

8. K. Horie, W. Schnabel, I. Mita, and H. Ushiki, Macromolecules, 14, 1422 (1981).

9. H. Ushiki, K. Horie, A. Okamoto, and I. Mita, Polym. J., 13, 191 (1981).

10. H. Ushiki, K. Hirayanagi, Y. Shindo, K. Horie, and I. Mita, Polym. J., 15, 811 (1983).

11. Y. Shindo, T. Sugimura, and K. Horie, Eur. Polym. J., 25, 1033 (1989).

12. Y. Shindo, T. Sugimura, K. Horie, and I. Mita, Polym. J., 22, 63 (1990).

13. Y. Shindo, T. Sugimura, K. Horie, and I. Mita, Eur. Polym. J., 26, 221 (1990).

14. T. Torii, H. Ushiki, and K. Horie, Polym. J., 24, 1057 (1992).

15. M. A. Winnik, Ed., "Photophysical and Photochemical Tools in Polymer Science," NATO ASI series C-182, D. Reidel, Dordrecht, Holland, 1986.

16. Z. Grubisic, P. Rempp, and H. Benoit, J. Polym. Sci., B, 5, 753 (1967).

17. P. J. Flory and T. G. Fox, J. Am. Chem. Soc., 73, 1904 (1951).

18. I. Mita and K. Horie, J. Macromol. Sci-Rev. Macromol. Chem. Phys., C27, 91 (1987).

19. P. J. Flory, J. Am. Chem. Soc., 61, 1518 (1939).

20. E. R. Cohen and H. Reiss, J. Chem. Phys., 38, 680 (1963). 